\title{
A Direct Approach to Area Change in Continuum Mechanics
}

COPPE/UFRJ - Programa de Engenharia

Mecânica

Cx. Postal 68503

21945-970 Rio de Janeiro, RJ. Brazil

Icm@serv.com.ufrj.br

\section{R. F. de Oliveira}

COPPE/UFRJ - Programa de Engenharia Civil

Cx. Postal 68519

21945-970 Rio de Janeiro, RJ. Brazil

roberto@coc.ufrj.br
In this note we use elementary facts from 3-dimensional linear algebra to demonstrate the formula for area changes under arbitrary linear maps $\mathbf{A}: \mathbf{R}^{3} \rightarrow \mathbf{R}^{3}$. We do it in general, from a hint given by the well known invertible case, which is an important result used in continuum mechanics. An intrinsic formula for the case when rank $\mathbf{A}=2$ is obtained and we finish by showing that we are really exploring $a$ property of the cofactor matrix of $\mathbf{A}$ for an orthonormal basis.

Keywords : Cofactor matrix, linear algebra

\section{Introduction}

In calculus courses it is standard to consider the lenght and volume changes corresponding to a mapping $f: \mathbf{R}^{3} \rightarrow \mathbf{R}^{3}$. As for the area change, not even the invertible case is usually considered. In this note we approach this matter directly, in the linear case. This is the starting point for generalization to arbitrary smooth functions. And this result is needed when analyzing the properties of the Piola-Kirchhoff stress tensor (Gurtin, 1981, p. 178 and ff., for example).

Let $\mathbf{A}$ be a linear mapping from $\mathrm{V}$ to $\mathrm{V}$, a 3-dimensional real vector space, endowed with an inner product $\mathbf{u} \bullet \mathbf{v}$ of vectors $\mathbf{u}, \mathbf{v} \in$ $\mathrm{V}$, and oriented by the vector product $\mathbf{u} \times \mathbf{v}$. Recall that the transpose $\mathbf{A}^{\mathbf{T}}$ of $\mathbf{A}$ is defined by the equality $\mathbf{A u} \bullet \mathbf{v}=\mathbf{u} \bullet \mathbf{A}^{\mathbf{T}} \mathbf{v}$, holding for all $\mathbf{u}, \mathbf{v} \in \mathrm{V}$. From the standard properties of the mixed product, $\mathbf{A u} \times \mathbf{A v} \bullet \mathbf{A} \mathbf{w}=(\operatorname{det} \mathbf{A}) \mathbf{u} \times \mathbf{v} \bullet \mathbf{w}$, and observing that $(\operatorname{det} \mathbf{A}) \mathbf{u} \times \mathbf{v} \bullet \mathbf{w}=(\operatorname{det} \mathbf{A}) \mathbf{A}^{-\mathbf{T}}(\mathbf{u} \times \mathbf{v}) \bullet \mathbf{A w}$, where $\mathbf{A}^{-\mathbf{T}}$ is the inverse of $\mathbf{A}^{\mathbf{T}}$, we get $\mathbf{A u} \times \mathbf{A v} \bullet \mathbf{A w}=(\operatorname{det} \mathbf{A}) \mathbf{A}^{-\mathbf{T}}(\mathbf{u} \times \mathbf{v}) \bullet \mathbf{A w}$, valid whenever $\mathbf{A}$ is invertible. As $\mathbf{w}$ is arbitrary, it follows that

$$
\mathbf{A} \mathbf{u} \times \mathbf{A v}=(\operatorname{det} \mathbf{A}) \mathbf{A}^{-\mathbf{T}}(\mathbf{u} \times \mathbf{v})
$$

This standard result is the starting point of our approach: $\mathbf{A u} \times$ Av is always given by a linear mapping acting on $(\mathbf{u} \times \mathbf{v})$, even when $\mathbf{A}$ is not invertible.

\section{The Direct Approach}

Recall that the vectors $\mathbf{u} \times \mathbf{v}$ and $\mathbf{A u} \times \mathbf{A v}$ can be seen as normals to $\operatorname{span}\{\mathbf{u}, \mathbf{v}\}$ and $\operatorname{span}\{\mathbf{A u}, \mathbf{A v}\}$ respectively. As the oriented area of the parallelogram of sides $\mathbf{u}$ and $\mathbf{v}$ can be defined by $\mathbf{u} \times \mathbf{v}$, and the factor for the area change is constant, we realize that if $\mathbf{u}^{\prime} \times \mathbf{v}^{\prime}=\mathbf{u} \times \mathbf{v}$, then $\mathbf{A} \mathbf{u}^{\prime} \times \mathbf{A} \mathbf{v}^{\prime}=\mathbf{A} \mathbf{u} \times \mathbf{A v}$. Thus it appears that we can consider a mapping $\mathbf{A}^{*}: \mathrm{V} \rightarrow \mathrm{V}$ defined by the formula

$$
\mathbf{A}^{*}(\mathbf{u} \times \mathbf{v})=\mathbf{A u} \times \mathbf{A v} .
$$

Technical Note accepted June, 2003. Technical Editor: Atila P. Silva Freire.
Now we show that (2) makes sense and that $\mathbf{A}^{*}$ is linear.

Let $\{\mathbf{u}, \mathbf{v}, \mathbf{w}, \mathbf{r}\}$ be a set of vectors in $\mathrm{V}$. If we develop the expression $(\mathbf{u} \times \mathbf{v}) \bullet(\mathbf{w} \times \mathbf{r})$, first remembering that $(\mathbf{u} \times \mathbf{v}) \bullet(\mathbf{w} \times$ $\mathbf{r})=\mathbf{u} \bullet \mathbf{v} \times(\mathbf{w} \times \mathbf{r})$ and then performing the triple vector product, we get the well known identity:

$$
(\mathbf{u} \times \mathbf{v}) \bullet(\mathbf{w} \times \mathbf{r})=(\mathbf{u} \bullet \mathbf{w})(\mathbf{v} \bullet \mathbf{r})-(\mathbf{u} \bullet \mathbf{r})(\mathbf{v} \bullet \mathbf{w}) .
$$

Now applying (3) to the list $\{\mathbf{A u}, \mathbf{A v}, \mathbf{w}, \mathbf{r}\}$ it follows that

$$
(\mathbf{A u} \times \mathbf{A v}) \bullet(\mathbf{w} \times \mathbf{r})=(\mathbf{u} \times \mathbf{v}) \bullet\left(\mathbf{A}^{\mathbf{T}} \mathbf{w} \times \mathbf{A}^{\mathbf{T}} \mathbf{r}\right) .
$$

Choose a positive orthonormal basis $\{\mathbf{e}, \mathbf{f}, \mathbf{g}\}$ for $\mathrm{V}$. For $\mathbf{A}$ a linear map on $\mathrm{V}$, we define $\mathbf{B e}:=\mathbf{A}^{\mathbf{T}} \mathbf{f} \times \mathbf{A}^{\mathbf{T}} \mathbf{g}, \mathbf{B f}:=\mathbf{A}^{\mathbf{T}} \mathbf{g} \times$ $\mathbf{A}^{\mathbf{T}} \mathbf{e}$ and $\mathbf{B g}:=\mathbf{A}^{\mathbf{T}} \mathbf{e} \times \mathbf{A}^{\mathbf{T}} \mathbf{f}$, and extend $\mathbf{B}$ by linearity to all $\mathbf{V}$. Then (4) gives

$$
(\mathbf{A u} \times \mathbf{A v}) \bullet(\mathbf{f} \times \mathbf{g})=(\mathbf{u} \times \mathbf{v}) \bullet\left(\mathbf{A}^{\mathbf{T}} \mathbf{f} \times \mathbf{A}^{\mathbf{T}} \mathbf{g}\right)=\mathbf{u} \times \mathbf{v} \bullet \mathbf{B e},
$$

or

$$
(\mathbf{A u} \times \mathbf{A v}) \bullet \mathbf{e}=\mathbf{B}^{\mathbf{T}}(\mathbf{u} \times \mathbf{v}) \bullet \mathbf{e} .
$$

Thus by cyclic permutation of (5) and (6) we conclude that

$$
\mathbf{A u} \times \mathbf{A v}=\mathbf{B}^{\mathbf{T}}(\mathbf{u} \times \mathbf{v}),
$$

and to any $\mathbf{A}$ linear we can associate a linear map, which we call $\mathbf{A}^{*}$, such that

$$
\mathbf{A u} \times \mathbf{A v}=: \mathbf{A}^{*}(\mathbf{u} \times \mathbf{v})
$$

holds for any vectors $\mathbf{u}, \mathbf{v} \in \mathrm{V}$. Observe that $\mathbf{A}^{*}$ is uniquely determined by $\mathbf{A}$, and writing (4) as

$$
\mathbf{A}^{*}(\mathbf{u} \times \mathbf{v}) \bullet(\mathbf{w} \times \mathbf{r})=(\mathbf{u} \times \mathbf{v}) \bullet\left(\mathbf{A}^{\mathbf{T}}\right)^{*}(\mathbf{w} \times \mathbf{r})
$$

we can conclude that $\left(\mathbf{A}^{*}\right)^{\mathbf{T}}=\left(\mathbf{A}^{\mathbf{T}}\right)^{*}$. Finally, as $(\operatorname{det} \mathbf{A}) \mathbf{u} \times \mathbf{v} \bullet \mathbf{w}$ $=\mathbf{A u} \times \mathbf{A v} \bullet \mathbf{A w}=\mathbf{A}^{*}(\mathbf{u} \times \mathbf{v}) \bullet \mathbf{A w}=\mathbf{A}^{\mathbf{T}} \mathbf{A}^{*}(\mathbf{u} \times \mathbf{v}) \bullet \mathbf{w}$ holds for any three vectors, 


$$
\mathbf{A}^{\mathbf{T}} \mathbf{A}^{*}=(\operatorname{det} \mathbf{A}) \mathbf{I}
$$

using the transpose, $\mathbf{A}\left(\mathbf{A}^{*}\right)^{\mathbf{T}}=\left(\operatorname{det} \mathbf{A}^{\mathbf{T}}\right) \mathbf{I}$, we have also

$$
\mathbf{A}^{*} \mathbf{A}^{\mathbf{T}}=(\operatorname{det} \mathbf{A}) \mathbf{I} \text {. }
$$

In the invertible case we already know that $\mathbf{A}^{*}=(\operatorname{det} \mathbf{A}) \mathbf{A}^{-\mathbf{T}}$ (Chadwick, 1976, Ciarlet, 1988, Greub, 1967, Gurtin, 1981) and, by Ciarlet, 1988, that $\mathbf{A}^{*}=\mathbf{0}$ (Greub, 1967, Sinkhorn, 1993) when $\operatorname{rank} \mathbf{A}<2$. If the rank of $\mathbf{A}$ is exactly 2 (Sinkhorn, 1993), the defining property (8) also shows that rank $\mathbf{A}^{*}=1$ and because the range of $\mathbf{A}^{*}$ is the orthogonal complement of the image of $\mathbf{A}$, if we suppose this 1-dimensional subspace generated by the unit vector $\mathbf{m}$, it follows that $\mathbf{A}^{*}=\mathbf{m} \otimes \mathbf{a}$ for some vector $\mathbf{a}$ (recall that $(\mathbf{c} \otimes$ $\mathbf{b}) \mathbf{u}=(\mathbf{b} \bullet \mathbf{u}) \mathbf{c}$, for all vectors $\mathbf{u}$, defines the tensor product $\mathbf{c} \otimes \mathbf{b})$.

Let $\mathbf{n}$ be a unit vector in the kernel of $\mathbf{A}$. Then

$$
\mathbf{0}=\mathbf{A u} \times \mathbf{A n}=\mathbf{A}^{*}(\mathbf{u} \times \mathbf{n})=[\mathbf{a} \bullet(\mathbf{u} \times \mathbf{n})] \mathbf{m}=[\mathbf{u} \bullet(\mathbf{n} \times \mathbf{a})] \mathbf{m}
$$

shows that $\mathbf{a}$ is parallel to $\mathbf{n}$. Thus $\mathbf{A}^{*}=\alpha \mathbf{m} \otimes \mathbf{n}$ for some nonzero constant $\alpha$. Now if the list $\{\mathbf{p}, \mathbf{q}, \mathbf{n}\}$ is a positive orthonormal basis for $\mathbf{V}$, as $\mathbf{A}+\alpha \mathbf{m} \otimes \mathbf{n}$ is invertible and

$\operatorname{det}(\mathbf{A}+\alpha \mathbf{m} \otimes \mathbf{n})=\alpha \operatorname{det}(\mathbf{A}+\mathbf{m} \otimes \mathbf{n})$, then

$(\mathbf{A}+\alpha \mathbf{m} \otimes \mathbf{n}) \mathbf{p} \times(\mathbf{A}+\alpha \mathbf{m} \otimes \mathbf{n}) \mathbf{q} \bullet(\mathbf{A}+\alpha \mathbf{m} \otimes \mathbf{n}) \mathbf{n}=\alpha \operatorname{det}(\mathbf{A}+$ $\mathbf{m} \otimes \mathbf{n})$,

or

$\alpha \operatorname{det}(\mathbf{A}+\mathbf{m} \otimes \mathbf{n})=\mathbf{A p} \times \mathbf{A q} \bullet(\alpha \mathbf{m})=\alpha \mathbf{A}^{*} \mathbf{n} \bullet \mathbf{m}=\alpha^{2}$,

shows that

$$
\mathbf{A}^{*}=[\operatorname{det}(\mathbf{A}+m \otimes n)](m \otimes n),
$$

which is an intrinsic formula for $\mathbf{A}^{*}$ if $\operatorname{rank} \mathbf{A}=2$.

Remark: recall that the derivative of $f(\mathbf{A})=\operatorname{det} \mathbf{A}$ is given by (Šilhavý, 1997)

$$
\partial_{\mathbf{A}} f(\mathbf{A})=(\operatorname{det} \mathbf{A}) \mathbf{A}^{-\mathrm{T}}=\mathbf{A}^{*},
$$

whenever $\mathbf{A}$ is invertible, where we have used the standard representation theorem for linear forms.

Let's call Inv the subset of all invertible linear mappings from $\mathrm{V}$ onto $\mathbf{V}$. Thus, if $\left\{\mathbf{A}_{\mathbf{n}}\right\}$ is a sequence of elements of $\mathbf{I n v}$ converging to $\mathbf{A}$,

$$
\mathbf{A}_{\mathrm{n}} \mathbf{u} \times \mathbf{A}_{\mathrm{n}} \mathbf{v}=\mathbf{A}_{\mathbf{n}}^{*}(\mathbf{u} \times \mathbf{v})
$$

shows that $\mathbf{A}_{\mathbf{n}}^{*}(\mathbf{u} \times \mathbf{v}) \rightarrow \mathbf{A u} \times \mathbf{A v}=: \mathbf{A}^{*}(\mathbf{u} \times \mathbf{v})$ and we conclude that $\mathbf{A}_{\mathbf{n}}^{*} \rightarrow \mathbf{A}^{*}$. Thus $\left\{\left(\operatorname{det} \mathbf{A}_{\mathrm{n}}\right) \mathbf{A}_{\mathrm{n}}^{-\mathrm{T}}\right\}$ converges to $\mathbf{A}^{*}$ whenever $\left\{\mathbf{A}_{\mathrm{n}}\right\}$ converges to $\mathbf{A}$, and we have also obtained a formula for the derivative of $f(\mathbf{A})=\operatorname{det} \mathbf{A}$ because we know that $\mathbf{I n v}$ is dense in the set of linear mappings from $\mathrm{V}$ into $\mathrm{V}$. $\left(\partial_{\mathrm{A}} f(\mathbf{A})\right.$ has entries which are polynomials in the entries of $\mathbf{A}$ ). In particular, if $\operatorname{rank} \mathbf{A}=2$, and as in (11) $\mathbf{m}$ is a unit vector orthogonal to the range of $\mathbf{A}$ and $\mathbf{n}$ is a unit vector in the kernel of $\mathbf{A}$,

$$
(\mathbf{A}+\mathbf{m} \otimes \mathbf{n})\left(\mathbf{A}^{\mathrm{T}}+\mathbf{n} \otimes \mathbf{m}\right)=\mathbf{A} \mathbf{A}^{\mathrm{T}}+\mathbf{m} \otimes \mathbf{m},
$$

where we have used the elementary facts that $(\mathbf{b} \otimes \mathbf{c})^{\mathrm{T}}=\mathbf{c} \otimes \mathbf{b}, \quad \mathbf{A}(\mathbf{b} \otimes \mathbf{c})=(\mathbf{A b}) \otimes \mathbf{c}, \quad(\mathbf{b} \otimes \mathbf{c}) \mathbf{A}=\mathbf{b} \otimes\left(\mathbf{A}^{\mathrm{T}} \mathbf{c}\right)$ and $(\mathbf{b} \otimes \mathbf{c})(\mathbf{d} \otimes \mathbf{e})=(\mathbf{c} \bullet \mathbf{d}) \mathbf{b} \otimes \mathbf{e}$.

We recall now that $\mathbf{A A}^{\mathrm{T}}$ is symmetric, invertible and positive when restricted to the range of $\mathbf{A}$. Let $\alpha^{2}$ and $\beta^{2}$ be the eigenvalues of $\mathbf{A} \mathbf{A}^{\mathrm{T}}$ ( $\alpha$ and $\beta$ are the non-negative proper values of $\mathbf{A}$ ). Thus the eigenvalues of $\left(\mathbf{A A}^{\mathrm{T}}+\mathbf{m} \otimes \mathbf{m}\right)$ being clearly $\alpha^{2}, \beta^{2}$ and 1, (14) shows that $(\operatorname{det}(\mathbf{A}+\mathbf{m} \otimes \mathbf{m}))^{2}=\alpha^{2} \beta^{2}$. Finally we observe that if we choose an orthonormal basis $(\mathbf{u}, \mathbf{v}, \mathbf{n})$ and $\mathbf{m}$ the unit vector in the sense of $\mathbf{A u} \times \mathbf{A v}$, we can construct a proper rotation $\mathbf{R}$ mapping the range of $\mathbf{A}^{\mathrm{T}}$ onto the range of $\mathbf{A}, \mathbf{n}$ into $\mathbf{R n}=\mathbf{m}$ and, moreover, for this choice we have $\operatorname{det}(\mathbf{A}+\mathbf{m} \otimes \mathbf{n})$ being positive and equals to $\alpha \beta$. Thus (11) can be written as

$$
\mathbf{A}^{*}=\alpha \beta \mathbf{R n} \otimes \mathbf{n},
$$

the well known standard formula for $\mathbf{A}^{*}$. Incidentally, that $\mathbf{A}^{*}=\mathbf{0}$ if $\operatorname{rank} \mathbf{A}<2$ also follows from (15).

Observe that if $\operatorname{rank} \mathbf{A}=2$ and $\mathbf{A}$ is symmetric (or skewsymmetric) then kernel of $\mathbf{A}$ is orthogonal to range of $\mathbf{A}$; if we choose $\mathbf{m}=\mathbf{n}$ we obtain directly from (11)

$$
\mathbf{S}^{*}=[\operatorname{det}(\mathbf{S}+\mathbf{n} \otimes \mathbf{n})](\mathbf{n} \otimes \mathbf{n})=\alpha \beta \mathbf{n} \otimes \mathbf{n},
$$

if $\mathbf{A}=\mathbf{S}$ is symmetric with nonzero eigenvalues $\alpha$ and $\beta$; and

$$
\mathbf{W}^{*}=[\operatorname{det}(\mathbf{W}+\mathbf{n} \otimes \mathbf{n})](\mathbf{n} \otimes \mathbf{n})=|\boldsymbol{\omega}|^{2} \mathbf{n} \otimes \mathbf{n}=\boldsymbol{\omega} \otimes \boldsymbol{\omega},
$$

if $\mathbf{A}=\mathbf{W}$ is skew-symmetric with $\boldsymbol{\omega}$ being its axial vector.

\section{Formulae Using Standard Basis.}

Finally in $\mathbf{R}^{3}$ we use the standard basis $\{\mathbf{i}, \mathbf{j}, \mathbf{k}\}$. The linear mapping $\mathbf{A}$ can be written as $\mathbf{A}=\mathbf{a} \otimes \mathbf{i}+\mathbf{b} \otimes \mathbf{j}+\mathbf{c} \otimes \mathbf{k}$, where $\mathbf{a}$, $\mathbf{b}$ and $\mathbf{c}$ are the images by $\mathbf{A}$ of $\mathbf{i}, \mathbf{j}$ and $\mathbf{k}$. Now it is clear that $\mathbf{A}^{*}=$ $(\mathbf{b} \times \mathbf{c}) \otimes \mathbf{i}+(\mathbf{c} \times \mathbf{a}) \otimes \mathbf{j}+(\mathbf{a} \times \mathbf{b}) \otimes \mathbf{k}$ because $\mathbf{a} \times \mathbf{b}=\mathbf{A} \mathbf{i} \times \mathbf{A j}=$ $\mathbf{A}^{*}(\mathbf{i} \times \mathbf{j})=\mathbf{A}^{*} \mathbf{k}$, and analogous computations for $\mathbf{A}^{*} \mathbf{i}$ and $\mathbf{A}^{*} \mathbf{j}$. Then the matrix of $\mathbf{A}^{*}$ is the cofactor matrix of $\mathbf{A}$. Now as

$\mathbf{A}^{*} \mathbf{A}^{\mathbf{T}}=(\mathbf{b} \times \mathbf{c}) \otimes \mathbf{a}+(\mathbf{c} \times \mathbf{a}) \otimes \mathbf{b}+(\mathbf{a} \times \mathbf{b}) \otimes \mathbf{c}=\mathbf{A}^{\mathbf{T}} \mathbf{A}^{*}=(\mathbf{a}$ $\bullet \mathbf{b} \times \mathbf{c}) \mathbf{I}$,

where we have used the well known property $(\mathbf{u} \otimes \mathbf{v})(\mathbf{w} \otimes \mathbf{r})=(\mathbf{v}$ $\bullet \mathbf{w})(\mathbf{u} \otimes \mathbf{r})$, it follows the identity

$$
(\mathbf{a} \bullet \mathbf{b} \times \mathbf{c}) \mathbf{v}=(\mathbf{a} \bullet \mathbf{v}) \mathbf{b} \times \mathbf{c}+(\mathbf{b} \bullet \mathbf{v}) \mathbf{c} \times \mathbf{a}+(\mathbf{c} \bullet \mathbf{v}) \mathbf{a} \times \mathbf{b} .
$$
is

Observe that if $\{\mathbf{a}, \mathbf{b}, \mathbf{c}\}$ is a basis for $\mathbf{R}^{3}$, its reciprocal basis

$$
\left\{\frac{\mathbf{b} \times \mathbf{c}}{\mathbf{a} \cdot \mathbf{b} \times \mathbf{c}}, \frac{\mathbf{c} \times \mathbf{a}}{\mathbf{a} \cdot \mathbf{b} \times \mathbf{c}}, \frac{\mathbf{a} \times \mathbf{b}}{\mathbf{a} \cdot \mathbf{b} \times \mathbf{c}}\right\} .
$$

\section{Acknowledgement}


We gratefully acknowledge the support of this work by CNPq, and the observations of a referee that inspired us to improve the proof of our main result, in a first draft of this work.

\section{References}

Chadwick, P., 1976, "Continuum Mechanics: concise theory and problems", George Allen and Unwin Ltd, London, U.K..
Ciarlet, P.G., 1988, "Mathematical Elasticity, vol I: Three-Dimensional Elasticity", North-Holland, Amsterdam, The Netherlands.

Greub, W.H., 1967, "Multilinear Algebra", Springer-Verlag, Berlin, Germany.

Gurtin, M.E., 1981, "An Introduction to Continuum Mechanics", Academic Press, New York, U.S.A..

Šilhavý, M., 1997, "The Mechanics and Thermodynamics of Continuous Media", Springer-Verlag, Berlin, Germany.

Sinkhorn, R., 1993, "The Range of the Adjugate Map", Mathematics Magazine, vol. 66, No. 2, 109-113. 
A Direct Approach to Area Change in Continuum Mechanics 